\title{
Complexity of Admissible Rules
}

\author{
Emil Jeřábek* \\ Mathematical Institute of AS CR \\ Žitná 25 \\ 11567 Praha 1 \\ Czech Republic \\ jerabek@math.cas.cz
}

September 19, 2006

\begin{abstract}
We investigate the computational complexity of deciding whether a given inference rule is admissible for some modal and superintuitionistic logics. We state a broad condition under which the admissibility problem is coNEXP-hard. We also show that admissibility in several well-known systems (including $G L, S 4$, and $I P C$ ) is in $c o N E$, thus obtaining a sharp complexity estimate for admissibility in these systems.
\end{abstract}

\section{Introduction}

Computational complexity of derivability in modal and superintuitionistic logics is a wellestablished subject. Kuznetsov [15] studied complexity of s.i. logics, and posed the problem whether $I P C$ is coNP-complete. Ladner [16] showed that the modal systems $K, T$, and $S 4$ are $P S P A C E$-complete, whereas $S 5$ is in coNP. Statman [20] refuted Kuznetsov's conjecture, by showing PSPACE-completeness of $I P C$, and even of its implicational fragment. Since then similar results were obtained for a variety of modal and s.i. logics, see e.g. Chagrov [3] and Spaan [19]; the bottom line is that PSPACE is the "typical" complexity of modal and s.i. logics with unbounded width and depth.

In contrast, the complexity of admissibility in non-classical logics is mostly unknown. An inference rule

$$
\frac{\varphi_{1}, \ldots, \varphi_{k}}{\psi}
$$

is admissible in a logic $L$, if the set of theorems of $L$ is closed under the rule. Friedman [7] asked whether admissibility in intuitionistic logic is decidable. The problem was extensively studied in the 80's and 90's in a series of papers by Rybakov, later summarized in the book [18]. Among other deep results on properties of sets of admissible rules and their bases,

${ }^{*}$ The research was done while the author was visiting the Department of Philosophy of the Utrecht University. Supported by grant IAA1019401 of GA AV ČR. 
Rybakov showed decidability of admissibility in many modal and s.i. logics (thus answering positively Friedman's question). Chagrov [4] constructed a decidable modal logic, which has undecidable admissibility problem. The work of Ghilardi on unification in intuitionistic and modal logics $[8,9]$ provided an important characterization of admissibility in terms of projective formulas, and new decision procedures for admissibility in some modal and s.i. systems. Ghilardi's results were utilized by Iemhoff $[11,12,13]$ to construct an explicit basis of admissible rules for $I P C$ and some other s.i. logics, and to develop Kripke semantics for admissible rules. These results were extended to modal logics by Jeřábek [14].

The study of algorithmic aspects of admissibility thus so far concentrated on the question of decidability. The complexity of some of the known decision procedures for admissibility is indicated in the literature, and we may compute estimates for the other ones with little effort. Namely, Rybakov (see [18]) gives a decision procedure for admissibility of reduced rules in $K 4, G L, S 4, S 4 G r z$, and $I P C$, which is easily seen to be implementable in $\Pi_{2}^{P}$. Admissibility of non-reduced rules in these systems is thus decidable in $\Pi_{2}^{E}=c o N E^{N P}$. Ghilardi [10] found a remarkably elegant algorithm for constructing projective approximations (and thus testing admissibility) in $I P C$, which appears more useful in practice, but makes a worse bound for theoretical purposes: exponential space. As we will see, these upper bounds turn out to be mostly suboptimal. More importantly, as far as the author is aware, no nontrivial lower bounds were known for the admissibility problem, except for Chagrov's example.

Our aim is to fill this gap by showing that admissibility in "typical" normal extensions of $K 4$ and s.i. logics is coNEXP-complete (and in particular, strictly more complex than the derivability problem, under reasonable complexity-theoretic assumptions). On one hand, we modify our algorithm from [14] to obtain a coNEXP (in fact, coNE) decision procedure for admissibility in a class of logics including $K 4, G L, S 4, S 4 G r z$, and IPC. On the other hand, we show that admissibility is coNEXP-hard in all s.i. $\operatorname{logics} L$ contained in $B D_{3}$ (i.e., every depth-3 tree is an $L$-frame), and in normal extensions of $K 4$ meeting a similar requirement.

\section{Preliminaries}

We assume the reader is familiar with basics of the theory of modal and superintuitionistic (s.i.) logics; we refer the reader to [5] or [1] for concepts unexplained here. We also assume rudimentary complexity theory (definitions of complexity classes such as the polynomial hierarchy, PSPACE, NE, and NEXP; consult e.g. [17]). Our results (especially the upper bounds) depend heavily on [11] and [14]; we summarize the relevant definitions and results below.

All Kripke frames are assumed to be transitive. Usually we will denote the accessibility relation by the ordering symbol $<$, in which case $\leq$ is its reflexivization: $x \leq y$ iff $x=y$ or $x<y$. (If the frame is already reflexive, we thus have $<=\leq$.) The symbols $\square^{n} \varphi, \triangleright \varphi, \nabla \varphi$, and $\diamond \varphi$ abbreviate $\underbrace{\square \cdots \square}_{n} \varphi, \varphi \wedge \square \varphi, \neg \square \neg \varphi$, and $\neg \square \neg \varphi$, respectively.

Some modal axioms, and their corresponding conditions on finite transitive Kripke frames, are listed in table 1 . We let $K$ be the minimal normal modal logic, $G L=K \oplus(G L)$, and $S 4=K \oplus(4) \oplus(T)$; names of other modal logics will be formed by concatenation of the name 


\begin{tabular}{|c|l|l|}
\hline symbol & axiom & frame condition \\
\hline$(4)$ & $\square \varphi \rightarrow \square \square \varphi$ & (transitive) \\
$(T)$ & $\square \varphi \rightarrow \varphi$ & reflexive \\
$(G L)$ & $\square(\square \varphi \rightarrow \varphi) \rightarrow \square \varphi$ & irreflexive \\
$(.1)$ & $\square \diamond \varphi \rightarrow \diamond \square \varphi$ & $\forall x \exists y \geq x \forall z(y<z \rightarrow y=z)$ \\
$($ Grz $)$ & $\square(\square(\varphi \rightarrow \square \varphi) \rightarrow \varphi) \rightarrow \square \varphi$ & antisymmetric \\
$(.3)$ & $\square(\square \varphi \rightarrow \psi) \vee \square(\square \psi \rightarrow \varphi)$ & trichotomic \\
\hline
\end{tabular}

Table 1: Modal axioms

of a base logic, and additional axioms: e.g., $K 4, S 4.3, K 4 G r z$.

Let $L$ be a normal modal or superintuitionistic logic. A multiple-conclusion rule consists of two finite sets of formulas

$$
\frac{\varphi_{1}, \ldots, \varphi_{k}}{\psi_{1}, \ldots, \psi_{\ell}} .
$$

Such a rule is $L$-admissible, which we write as $\varphi_{1}, \ldots, \varphi_{k} \sim_{L} \psi_{1}, \ldots, \psi_{\ell}$, if for every substitution $\vec{\chi}$ : if $\vdash_{L} \varphi_{i}(\vec{\chi})$ for each $i$, then $\vdash_{L} \psi_{j}(\vec{\chi})$ for some $j$. The rule is $L$-derivable, if $\varphi_{1}, \ldots, \varphi_{k} \vdash_{L} \psi_{j}$ for some $j$. (In the modal case, derivation from a set of assumptions allows the necessitation rule, thus $\varphi \vdash_{L} \square \varphi$.)

A rule system over $L$ is a set $A$ of rules (written in a sequent form $\Gamma \triangleright \Delta$, where $\Gamma$ and $\Delta$ are finite sets of formulas), which is closed under cut, and includes all $L$-derivable rules. By abuse of language, we identify $L$ with the minimal rule system over $L$. The set of all $L$-admissible multiple-conclusion rules forms a rule system, which we denote $A_{L}$.

A quasi-normal modal logic is an extension of $K$ closed under substitution and detachment. Let $L$ be a normal extension of $K 4$. The characteristic formula of a rule $\Gamma \triangleright \Delta$ is

$$
\bigwedge_{\varphi \in \Gamma} \square \varphi \rightarrow \bigvee_{\psi \in \Delta} \square \psi
$$

If $A$ is a rule system over $L$, we let $A^{\square}$ denote the quasi-normal extension of $K 4$ axiomatized by characteristic formulas of all rules from $A$. In this way, we embed rule systems in quasinormal logics; the following observation ensures the embedding is faithful.

Lemma 1.1 ([14]) $A^{\square}$ is conservative over $A$, i.e., a sequent $\Gamma \triangleright \Delta$ is provable in $A$ if and only if its characteristic formula is provable in $A^{\square}$.

In particular, the study of $L$-admissible rules is subsumed by the study of $A_{L}^{\square}$. Also notice that the logic of the minimal rule system, $L^{\square}=K 4+\left\{\square \varphi ; \vdash_{L} \varphi\right\}$, is normal. It has the following semantical characterization.

Lemma 1.2 ([14]) Let $L$ be a Kripke complete normal extension of $K 4$. Then $L^{\square}$ is sound and complete with respect to the class of transitive rooted frames $\langle K,<, r\rangle$ such that $r$ is irreflexive, and $K \backslash\{r\}$ is an $L$-frame. If $L$ has the finite model property, then $L^{\square}$ has FMP as well. 
Let $L$ be a normal extension of $K 4$ with FMP. $L$ is reflexive, if all $L$-frames are reflexive (i.e., $L \supseteq S 4$ ), and it is irreflexive if all $L$-frames are irreflexive (i.e., $L \supseteq G L$ ). $L$ is linear if all rooted $L$-frames are linear (i.e., $L \supseteq K 4$.3). If $K_{i}, i<n$ are frames, then $\sum_{i<n} K_{i}$ is their disjoint sum. If $K$ is a frame, then $K^{\circ}\left(K^{\bullet}\right)$ is the frame constructed from $K$ by attaching a new reflexive (irreflexive) root below $K . L$ is extensible, if for every finite sequence of finite $L$-frames $K_{i}, i<n$, we have

- $\left(\sum_{i<n} K_{i}\right)^{\circ}$ is an $L$-frame, unless $L$ is irreflexive,

- $\left(\sum_{i<n} K_{i}\right)^{\bullet}$ is an $L$-frame, unless $L$ is reflexive.

$L$ is linear extensible, if it is linear, and satisfies the extensibility condition above for $n \leq 1$.

We introduce the following rule systems:

$$
\begin{aligned}
\square \varphi & \rightarrow \bigvee_{i<n} \square \psi_{i} \triangleright\left\{\boxminus \varphi \rightarrow \psi_{i} ; i<n\right\} \\
\bigwedge_{j<m}\left(\varphi_{j} \equiv \square \varphi_{j}\right) & \rightarrow \bigvee_{i<n} \square \psi_{i} \triangleright\left\{\bigwedge_{j<m} \square \varphi_{j} \rightarrow \psi_{i} ; i<n\right\}
\end{aligned}
$$

where $n, m \in \omega$. Let $A^{\circ, 1}$ and $A^{\bullet, 1}$ be the restrictions of $A^{\circ}$ and $A^{\bullet}$ to $n \leq 1$. The main theorem of [14] was the following description of admissible rules of extensible and linearly extensible modal logics.

Theorem 1.3 ([14]) If $L$ is an extensible modal logic, then L-admissible multiple-conclusion rules have a basis consisting of

- $A^{\bullet}$, unless $L$ is reflexive,

- $A^{\circ}$, unless $L$ is irreflexive.

The same holds for linear extensible logics, with $A^{\bullet, 1}$ and $A^{\circ, 1}$ in place of $A^{\bullet}$ and $A^{\circ}$.

Let $\langle K,<\rangle$ be an $L$-frame. A point $x$ is an irreflexive tight predecessor of a finite set of points $\left\{y_{i} ; i<n\right\}$, if for every $z \in K$,

$$
z>x \quad \text { iff } \quad \exists i z \geq y_{i} .
$$

$x$ is a reflexive tight predecessor of $\left\{y_{i} ; i<n\right\}$, if for every $z \in K$,

$$
z>x \quad \text { iff } \quad z=x \vee \exists i z \geq y_{i} .
$$

An $L$-frame $\langle K,<\rangle$ is extensible (resp., linearly extensible), provided the generated submodels $K_{x}$ are finite for every $x \in K$, and every finite subset of $K$ (resp., every subset of size at most 1 ) has a reflexive tight predecessor unless $L$ is irreflexive, and an irreflexive tight predecessor unless $L$ is reflexive.

Notice that an irreflexive tight predecessor may actually be a reflexive point: specifically, if $y$ is reflexive, than $y$ itself is both a reflexive t.p. and an irreflexive t.p. of $\{y\}$. We deviated from the definition in [14] in this respect ${ }^{1}$.

\footnotetext{
${ }^{1}$ More precisely, from its intended meaning; the definition of tight predecessors as it stands in [14] is erroneous.
} 
Let $L$ be an extensible logic, and $\varphi$ a formula. We define $R_{\varphi}^{L}$ as the conjunction of the formulas of the form

$$
\square\left(\bigwedge_{j<m} \square \varphi_{j} \rightarrow \bigvee_{i<n} \square \psi_{i}\right) \rightarrow \bigvee_{i<n} \square\left(\bigwedge_{j<m} \boxminus \varphi_{j} \rightarrow \psi_{i}\right)
$$

if $L$ is not reflexive, and

$$
\square\left(\bigwedge_{j<m}\left(\varphi_{j} \equiv \square \varphi_{j}\right) \rightarrow \bigvee_{i<n} \square \psi_{i}\right) \rightarrow \bigvee_{i<n} \square\left(\bigwedge_{j<m} \square \varphi_{j} \rightarrow \psi_{i}\right)
$$

if $L$ is not irreflexive, where $\square \varphi_{j}$ and $\square \psi_{i}$ are subformulas of $\varphi$.

Similarly, if $L$ is a linear extensible logic, we let $R_{\varphi}^{L}$ be the conjunction of

$$
\begin{gathered}
\diamond \bigwedge_{j<m} \square \varphi_{j}, \\
\square\left(\bigwedge_{j<m} \square \varphi_{j} \rightarrow \bigvee_{i<n} \square \psi_{i}\right) \rightarrow \square\left(\bigwedge_{j<m} \sqcup \varphi_{j} \rightarrow \bigvee_{i<n} \sqcup \psi_{i}\right),
\end{gathered}
$$

for $L$ not reflexive, and

$$
\begin{gathered}
\diamond \bigwedge_{j<m}\left(\varphi_{j} \equiv \square \varphi_{j}\right), \\
\square\left(\bigwedge_{j<m}\left(\varphi_{j} \equiv \square \varphi_{j}\right) \rightarrow \bigvee_{i<n} \square \psi_{i}\right) \rightarrow \square\left(\bigwedge_{j<m} \square \varphi_{j} \rightarrow \bigvee_{i<n} \square \psi_{i}\right),
\end{gathered}
$$

for $L$ not irreflexive, where $\square \varphi_{j}$ and $\square \psi_{i}$ are subformulas of $\varphi$. Notice that in both cases, $R_{\varphi}^{L}$ is (a simple variant of) a conjunction of axioms of $A_{L}^{\square}$. We have the following semantical characterization of $A_{L}^{\square}$ (and thus, of $L$-admissibility).

Theorem 1.4 ([14]) If $L$ is an extensible (linear extensible) modal logic, and $\varphi$ a formula, the following are equivalent.

(i) $A_{L}^{\square} \vdash \varphi$,

(ii) $L^{\square} \vdash R_{\varphi}^{L} \rightarrow \varphi$,

(iii) $\varphi$ holds in the root of every $L^{\square}$-frame $\langle K,<, r\rangle$ such that $K \backslash\{r\}$ is extensible (resp., linearly extensible).

In particular, the formulas $R_{\varphi}^{L}$ hold in the root of every $L^{\square}$-frame $K$ such that $K \backslash\{r\}$ is (linearly) extensible.

Earlier, R. Iemhoff [11] characterized the admissible rules of $I P C$. The main results (reformulated for multiple-conclusion rules) are as follows.

Theorem 1.5 ([11]) Visser's rules

$$
\bigwedge_{i<n}\left(\varphi_{i} \rightarrow \psi_{i}\right) \rightarrow \varphi_{n} \vee \varphi_{n+1} \triangleright\left\{\bigwedge_{i<n}\left(\varphi_{i} \rightarrow \psi_{i}\right) \rightarrow \varphi_{j} ; j \leq n+1\right\}
$$

together with the rule $\perp \triangleright$ form a basis of IPC-admissible rules. 
An intuitionistic frame $K$ is extensible, if every finite subset of $K$ has a reflexive tight predecessor.

Theorem 1.6 ([11]) A rule $\Gamma \triangleright \Delta$ is IPC-admissible if and only if every extensible model which satisfies $\Gamma$ also satisfies some formula $\psi \in \Delta$.

A frame $\left\langle K^{\prime},<^{\prime}\right\rangle$ is a subframe of a Kripke frame $\langle K,<\rangle$, if $K^{\prime}$ is a subset of $K$, and $<^{\prime}$ is the restriction of $<$ to $K^{\prime} . K^{\prime}$ is a cofinal subframe if additionally

$$
x \in K^{\prime} \wedge x<y \Rightarrow \exists z \in K^{\prime} y \leq z
$$

holds for every $x, y \in K$. A normal extension of $K 4$ is a subframe $(S F)$ logic, if it is complete with respect to a class of Kripke frames closed under subframes, and it is a cofinal subframe $(C S F)$ logic, if it is complete with respect to a class of Kripke ${ }^{2}$ frames closed under cofinal subframes. All cofinal subframe logics have the finite model property (Zakharyaschev [21], cf. [5]). CSF includes the vast majority of transitive logics used in practice: for example, logics axiomatized by combinations of the axioms listed in table 1 are CSF (in fact, SF, with the exception of (.1)).

If $K$ is a frame, the depth $d(x)$ of a point $x \in K$ is the maximal natural number $n$ such that there exists a sequence $x=x_{1}<x_{2}<\cdots<x_{n}$ in $K$ such that $x_{i+1} \nless x_{i}$. If the maximum does not exist, $d(x)=\infty$. The depth of a frame $K$ is $\sup \{d(x) ; x \in K\}$. The s.i. $\operatorname{logic} B D_{n}$ is defined as the set of formulas valid in all Kripke frames of depth at most $n$.

\section{Upper bounds}

In this section we aim to show that admissibility in some of the best-known transitive logics is decidable in coNEXP. We will use a modification of an exponential-space algorithm for admissibility described in Jeřábek [14]. The main idea is to show that the formulas $R_{\varphi}^{L} \rightarrow \varphi$ have a kind of exponential model property. Notice that this is nontrivial: in our logics a formula may have only models of exponential size, and the length of $R_{\varphi}^{L} \rightarrow \varphi$ is itself exponential in the length of $\varphi$, thus a priori it could require models of doubly exponential size.

We stress that we are only concerned about the theoretical complexity of admissibility. For practical purposes, our algorithm is no better than the earlier exponential-space and doubly-exponential time algorithms, as non-deterministic Turing machines do not exist in the real world. In particular, the algorithm of Ghilardi [10] is likely far more efficient in practice.

Definition 2.1 Let $\langle K,<, \Vdash\rangle$ be a finite model, $K^{\prime}$ its submodel, and $S$ a set of formulas. The submodel $K^{\prime}$ is $S$-faithful, if

$$
K, u \Vdash \psi \Leftrightarrow K^{\prime}, u \Vdash \psi
$$

for every $\psi \in S$ and $u \in K^{\prime}$.

\footnotetext{
${ }^{2}$ The usual definition of (cofinal) subframe logics is more complicated, and in particular, Kripke completeness of (C)SF logics is a nontrivial theorem rather than part of the definition. We ignore such subtleties.
} 
Lemma 2.2 Let $L$ be an extensible cofinal subframe logic. If $A_{L}^{\square} \nvdash \varphi$, there exists a rooted $L^{\square}{ }_{-m o d e l}\langle K,<, r, \Vdash\rangle$ of size $2^{O(n)}$ such that $r \Vdash R_{\varphi}^{L} \wedge \neg \varphi$, where $n$ is the length of $\varphi$.

Proof: As CSF logics have the finite model property, there exists a finite rooted $L^{\square}$-model $\langle K,<, r, \Vdash\rangle$ such that $r \Vdash R_{\varphi}^{L} \wedge \neg \varphi$. Let $S$ be the set of subformulas of $\varphi$, and $B$ the set of formulas $\beta$ such that $\square \beta$ is a subformula of $R_{\varphi}^{L}$. For any $\beta \in B$ such that $r \nVdash \square \beta$, we pick $x_{\beta} \in K$ such that $x_{\beta} \nVdash \beta$. By the proof of theorem 4.3 in Zakharyaschev [21], there exists an $S$-faithful submodel $K_{\beta} \subseteq K$ of size $2^{O(n)}$ such that $x_{\beta} \in K_{\beta}$. Let $K^{\prime}$ be the union of all $K_{\beta}$ and $\{r\}$. As $|B|=2^{O(n)}$, we have $\left|K^{\prime}\right|=2^{O(n)}$. The model $K^{\prime}$ is $S$-faithful, and as all formulas from $B$ are Boolean combinations of formulas from $S$, it is also $B$-faithful. It follows that $K$ and $K^{\prime}$ agree on satisfaction of boxed subformulas of $R_{\varphi}^{L}$ (and $\varphi$ ) in $r$, thus $\left\langle K^{\prime}, r\right\rangle \Vdash R_{\varphi}^{L} \wedge \neg \varphi$.

If $L$ is a subframe logic, then $K^{\prime}$ is an $L^{\square}$-frame, as $K^{\prime} \backslash\{r\}$ is a subframe of $K \backslash\{r\}$. If $L$ is only CSF, we have to modify $K^{\prime}$ further. For any cluster $C$, let $v(C)=\{\psi ; \square \psi \in S, x \nVdash \square \psi\}$, where $x \in C$ (the definition is independent on the choice of $x$ ). Let $X$ be the set of all final clusters $C \subseteq K$ such that $C \cap K^{\prime}=\emptyset$. We consider the following equivalence relation on $X$ : $C \sim D$ iff $v(C)=v(D)$, and either both $C$ and $D$ are reflexive, or both are irreflexive. Notice that $\sim$ has at most $2^{n}$ equivalence classes. Let $Y$ be a selector for $\sim$, and for every $C \in Y$, we pick a subset $C^{\prime} \subseteq C$ of size at most $n$ such that for every $\psi \in v(C)$, there exists an $x \in C^{\prime}$ such that $x \nVdash \psi$. We put $K^{\prime \prime}=K^{\prime} \cup \bigcup_{C \in Y} C^{\prime}$, and

$$
x \prec y \Leftrightarrow x<y \vee \exists C \in X \exists D \in Y\left(x<C \wedge C \sim D \wedge y \in D^{\prime}\right) .
$$

It is easy to see that satisfaction of subformulas of $\varphi$ is still preserved in $\left\langle K^{\prime \prime}, \prec, r, \Vdash\right\rangle$, thus $r \Vdash R_{\varphi}^{L} \wedge \neg \varphi$ in $K^{\prime \prime}$ by the same argument as above. The size of $K^{\prime \prime}$ is $2^{O(n)}$, and $K^{\prime \prime}$ is an $L^{\square}$-frame, as $\left\langle K^{\prime \prime} \backslash\{r\}, \prec\right\rangle$ is a p-morphic image of a cofinal subframe of $\langle K \backslash\{r\},<\rangle$.

Theorem 2.3 If $L$ is an extensible finitely axiomatizable CSF logic, then $A_{L}^{\square}$ (and thus $L$ admissibility of multiple-conclusion rules) is decidable in coNE. The same holds for admissibility in IPC.

Proof: Notice that it is decidable in polynomial time (in fact, in uniform $A C^{0}$ ) whether a finite Kripke frame is an $L$-frame, because CSF logics are elementary on finite frames [21]. To check that $A_{L}^{\square} \nvdash \varphi$, guess an exponential-size rooted model $\langle K,<, r, \Vdash\rangle$ together with valuation of all subformulas of $\varphi$, and verify that the valuation satisfies the usual inductive definition, $r \Vdash R_{\varphi}^{L} \wedge \neg \varphi$, and $\langle K \backslash\{r\},<\rangle$ is an $L$-frame.

Gödel's translation of any s.i. logic in its largest modal companion is faithful wrt admissible rules (Rybakov, see [18]). (Alternatively, in the case of $I P C$, it also follows from theorems 1.6 and 1.4.)

Example 2.4 Admissibility in $K 4, G L, S 4, K 4 G r z, S 4 G r z, K 4.1$, or $S 4.1$ is in coNE.

As noted in [14], admissibility in normal extensions of $G L .3$ or $S 4.3$ is coNP-complete. We expand this result to some other linear modal logics, including $K 4.3, K 4 G r z .3$, and $K 4.1 .3$. 
Lemma 2.5 Let $L$ be a CSF linear extensible logic, which does not contain $S 4$ or $G L$. If $A_{L}^{\square} \nvdash \varphi$, there exists a polynomial-size rooted $L^{\square}$-model $\langle K,<, r, \Vdash\rangle$ such that $r \Vdash R_{\varphi}^{L} \wedge \neg \varphi$.

Proof: Let $S$ be the set of subformulas of $\varphi$, and we call a point $x \varphi$-reflexive if $x \Vdash \square \psi$ implies $x \Vdash \psi$ for every $\square \psi \in S$. We take a linearly extensible $L^{\square}$-model $\langle K,<, r, \Vdash\rangle$ such that $r \nVdash \varphi$.

First we find a polynomial-size submodel $X_{0} \subseteq K^{-}=K \backslash\{r\}$ such that $X_{0}$ contains a dead end and a simple reflexive final cluster (i.e., t.p.'s of the empty set), $X_{0} \cup\{r\}$ is an $S$-faithful submodel of $K$, and $X_{0}$ hits every final cluster which is above a point of $X_{0}$. We can construct $X_{0}$ as follows. We pick a dead end $x^{\bullet} \in K^{-}$, and a simple reflexive final point $x^{\circ} \in K^{-}$. For each formula $\psi$ such that $\square \psi \in S$, and $r \nVdash \square \psi$, we pick $x_{\psi}>r$ such that $x_{\psi} \nVdash \psi$, and $y_{\psi} \geq x_{\psi}$ which belongs to a final cluster of $K^{-}$(there is only one final cluster above $x_{\psi}$, by linearity). For each $\square \chi \in S$ such that $x_{\psi} \nVdash \square \chi$, we find $z_{\psi, \chi}>x_{\psi}$ such that $z_{\psi, \chi} \nVdash \chi$, and $\chi$ holds in all points strictly above the cluster of $z_{\psi, \chi}$. We define $X_{0}$ as the set of all $x_{\psi}, y_{\psi}$, and $z_{\psi, \chi}$, together with $x^{\circ}$ and $x^{\bullet}$. Clearly $\left|X_{0}\right|=O\left(n^{2}\right)$, where $n=|\varphi|$. If $\square \omega \in S$ and $z_{\psi, \chi} \nVdash \square \omega$, then $z_{\psi, \chi}<z_{\psi, \omega} \nVdash \omega$ by linearity and the choice of $z_{\psi, \omega}$. This implies that $X_{0} \subseteq K^{-}$is $S$-faithful, and the other properties of $X_{0}$ are obvious.

We define a sequence $X_{1}, X_{2}, \ldots, X_{n}$ of subsets of $K^{-}$as follows: for any $x \in X_{i}$ which is not $\varphi$-reflexive, we pick a reflexive and an irreflexive t.p. of $\{x\}$ in $K^{-}$, and put them in $X_{i+1}$. Notice that $\left|X_{i+2}\right| \leq\left|X_{i+1}\right|$ as reflexive points are $\varphi$-reflexive, therefore $\left|X_{i}\right| \leq\left|X_{1}\right| \leq 2\left|X_{0}\right|=O\left(n^{2}\right)$. We claim that $X_{n}=\emptyset$. If not, then by the construction there exists a chain $x_{n}<x_{n-1}<\cdots<x_{1}<x_{0}$ such that $x_{i} \in X_{i}$, and $x_{i+1}$ is an irreflexive t.p. of $x_{i}$. A formula of the form $\square \psi \rightarrow \psi$ can fail in at most one point of a chain. As $|\{\psi ; \square \psi \in S\}|<n$, the pigeonhole principle implies that $x_{i}$ is $\varphi$-reflexive for some $i<n$, contradicting the definition of $X_{i+1}$.

We put $K^{\prime}=\{r\} \cup \bigcup_{i<n} X_{i}$. We have $\left|K^{\prime}\right|=O\left(n^{3}\right)$, and $K^{\prime}$ is an $L^{\square}$-model, as $K^{\prime} \backslash\{r\}$ is a cofinal submodel of $K^{-}$. It is easy to see that $K^{\prime}$ is an $S$-faithful submodel of $K$, in particular $r \nVdash \varphi$ in $K^{\prime}$. We claim that $r \Vdash R_{\varphi}^{L}$ in $K^{\prime}$. Consider for instance the formula

$$
\square\left(\bigwedge_{j<m} \square \varphi_{j} \rightarrow \bigvee_{i<n} \square \psi_{i}\right) \rightarrow \square\left(\bigwedge_{j<m} \boxminus \varphi_{j} \rightarrow \bigvee_{i<n} \square \psi_{i}\right),
$$

where $\square \varphi_{j}$ and $\square \psi_{i}$ are subformulas of $\varphi$. Assume that $r \nVdash \square\left(\bigwedge_{j<m} \boxminus \varphi_{j} \rightarrow \bigvee_{i<n} \boxminus \psi_{i}\right)$, and fix $x>r$ such that $x \Vdash \bigwedge_{j<m} \sqcup \varphi_{j} \wedge \bigwedge_{i<n} \neg \sqcup \psi_{i}$. If $x$ is not $\varphi$-reflexive, there exists an irreflexive t.p. $y>r$ of $\{x\}$ by the construction, and clearly $y \Vdash \bigwedge_{j<m} \square \varphi_{j} \wedge \bigwedge_{i<n} \neg \square \psi_{i}$. If $x$ is $\varphi$-reflexive, then trivially $x \Vdash \bigwedge_{j<m} \square \varphi_{j}$, and by $\varphi$-reflexivity $x \Vdash \bigwedge_{i<n} \neg \square \psi_{i}$. In both cases $r \nVdash \square\left(\bigwedge_{j<m} \square \varphi_{j} \rightarrow \bigvee_{i<n} \square \psi_{i}\right)$. The other conjuncts of $R_{\varphi}^{L}$ can be verified in a similar way.

Theorem 2.6 For any finitely axiomatizable linear extensible CSF logic L, admissibility and $A_{L}^{\square}$ are coNP-complete.

Proof: We may assume that $L$ does not contain $S 4$ or $G L$. We proceed similarly to the proof of theorem 2.3. If we guess a polynomial-size model of $\neg \varphi$ and verify it satisfies $R_{\varphi}^{L}$, we only get a $\Pi_{2}^{P}$-algorithm, as checking the exponential-size formula $R_{\varphi}^{L}$ consumes a quantifier. 
However, the proof of lemma 2.5 shows that we can assume the model to satisfy a stronger condition: every $x>r$ which is not $\varphi$-reflexive has a reflexive and an irreflexive t.p. This condition is checkable in polynomial time.

The usefulness of the next example will become clear later.

Proposition 2.7 For $L=G L+\square^{2} \perp, A_{L}^{\square}$ is in $\Pi_{3}^{P}$.

Proof: If $A_{L}^{\square} \nvdash \varphi$, there is an $A_{L}^{\square}$-model $K$ of $\neg \varphi$ with $O\left(n^{2}\right)$ leaves: take an arbitrary countermodel for $\varphi$, extract its polynomial-size submodel faithful wrt subformulas of $\varphi$, and augment it with all the missing tight predecessors (which will all have depth 2).

Existence of such a model is checkable in $\Sigma_{3}^{P}$ as follows. Find (first quantifier, $\exists$ ) a polynomial-size model $\langle K, r\rangle$ of depth 3 such that $r \nVdash \varphi$. For any nonempty set $X$ of leaves of $K$ (second quantifier, $\forall$ ), guess (third quantifier, $\exists$ ) a valuation of atoms in an imaginary tight predecessor $x$ of $X$, and verify the choice does not mess up satisfaction of subformulas of $\varphi$ in $r$ (i.e., for every boxed subformula $\square \psi$ of $\varphi$, if $r \Vdash \square \psi$ in $K$, then $x \Vdash \psi$ ).

\section{Lower bounds}

We are going to show that admissibility in a rich class of s.i. and modal logics is coNEXP-hard. We start by isolating a convenient $c o N E X P$-complete problem, which we will then reduce to admissibility.

Lemma 3.1 The following problem is NEXP-complete. Given a number $n$ in unary, and a sentence $\Phi$, determine whether $\Phi$ holds in the $n$-element structure (with no predicates or functions), where $\Phi$ is a $\Sigma_{1}^{2}$-formula of the form

$$
\exists X \forall a_{1} \ldots \forall a_{k} \varphi(X, \vec{a}, \vec{i})
$$

$X$ is a monadic third-order variable, $a_{j}$ are monadic second-order variables, $i_{j}$ are (firstorder) constants from the structure, and $\varphi$ is open.

Proof: Let $L$ be an $N E X P$-language. Identify a binary string $w$ with the structure $\langle n,<, W\rangle$, where $n=|w|$, and $W$ is the unary predicate such that $W(j)$ iff $w_{j}=1$. Standard encoding of Turing machine computations à la Fagin's theorem [6] gives a $\Sigma_{1}^{2}$-formula $\Phi$ such that

$$
w \in L \quad \text { iff } \quad w \vDash \Phi
$$

for every $w$. By usual quantifier switching tricks, any $\Sigma_{1}^{2}$-formula is equivalent to a formula of the form

$$
\exists \vec{X} \forall \vec{a} \varphi,
$$

where $\vec{X}, \vec{a}$, and $\varphi$ are third, second, and first order, respectively. By padding with unused places, we may also assume all $\vec{X}$ and $\vec{a}$ to have the same arity.

Let $w$ be given. We can tranform $\varphi$ into a polynomial-size quantifier-free formula using constants from $n$ instead of the relations $<, W$. By switching from $n$ to $n^{\prime}=n^{d}$ and 
adjusting the atomic subformulas of $\varphi$, we may assume that $\vec{a}$ are monadic. (Notice that the quantifier prefix is still constant at this point.) Assume that the existential prefix consists of $d$ quantifiers $\exists X_{j}$ of arity $c$. We can encode these $d c$-ary relations on subsets of $n^{\prime}$ by a single unary predicate on subsets of $n^{\prime \prime}=c n^{\prime}+|d|$ so that (in a sloppy notation) $X_{j}\left(a_{1}, \ldots, a_{c}\right)$ iff $X\left(\left\langle a_{1}, \ldots, a_{k}, j\right\rangle\right)$. We thus enlarge the structure once again from $n^{\prime}$ to $n^{\prime \prime}$, and replace the existential prefix with a single monadic quantifier. We retain the old quantifiers $\forall a_{j}$ (which now range over subsets of $n^{\prime \prime}$, representing the old subsets of $n^{\prime}$ by, say, the first $n^{\prime}$ coordinates), and for each atomic formula of $\varphi$ of the form $X_{j}(\vec{a})$, include a new quantifier $\forall b$ in the formula. In $\varphi$, replace $X_{j}(\vec{a})$ with $X(b)$, and include conditions (FO, rewritten as polynomial-size open) ensuring that $b$ is correctly formed from $\vec{a}$ and $j$.

The next theorem is a special case of theorem 3.13. We nevertheless prefer to include its proof separately, as it explains the intuition behind the construction used in the general case, without the complications necessary to deal with reflexive models and weaker expressive power of intuitionistic logic.

Theorem 3.2 Admissibility in $G L+\square^{3} \perp$ and $G L$ is coNEXP-hard.

Proof: Let $L=G L+\square^{3} \perp$. Assume we are given $n$, and a formula

$$
\Phi=\exists X \forall a_{0} \ldots \forall a_{k-1} \varphi\left(\ldots, a_{j} \in X, \ldots, i \in a_{j}, \ldots\right)
$$

as in lemma 3.1 , we will construct a formula $\bar{\Phi}$ such that

$$
n \vDash \Phi \quad \text { iff } \quad \bar{\Phi} \text { is } A_{L}^{\square} \text {-consistent. }
$$

We define $\bar{\Phi}$ as the conjunction of the following formulas in variables $p_{i}(i<n), q_{j}(j<k)$, and $t$ :

$$
\begin{gathered}
\square\left(\bigwedge_{i<i^{\prime}}\left(p_{i} \rightarrow \neg p_{i^{\prime}}\right) \wedge \bigwedge_{j<j^{\prime}}\left(q_{j} \rightarrow \neg q_{j^{\prime}}\right) \wedge \bigwedge_{i, j}\left(p_{i} \rightarrow \neg q_{j}\right)\right) \\
\square\left(\square \perp \equiv \bigvee_{i} p_{i} \vee \bigvee_{j} q_{j}\right) \\
\diamond\left(\bigwedge_{i} \diamond p_{i} \wedge \bigwedge_{j} \nabla q_{j}\right) \\
\square\left(\bigwedge_{i}\left(\square\left(\diamond \top \rightarrow \diamond p_{i}\right) \vee \square \neg \diamond p_{i}\right) \rightarrow \square(\diamond \top \rightarrow t) \vee \square(\diamond \top \rightarrow \neg t)\right) \\
\square\left(\bigwedge_{i, j}\left(\square\left(\diamond q_{j} \rightarrow \diamond p_{i}\right) \vee \square\left(\diamond q_{j} \rightarrow \neg \diamond p_{i}\right)\right) \wedge \bigwedge_{j} \diamond \diamond q_{j} \rightarrow \bar{\varphi}\right),
\end{gathered}
$$

where $\bar{\varphi}$ is the formula

$$
\varphi\left(\ldots, \diamond\left(t \wedge \diamond q_{j}\right), \ldots, \diamond\left(\diamond p_{i} \wedge \diamond q_{j}\right), \ldots\right)
$$

(Notice that $\neg \bar{\Phi}$ is a characteristic formula of a single-conclusion inference rule, thus we indeed reduce the problem to $L$-admissibility rather than full $A_{L}^{\square}$.) Recall from [14] that $A_{L}^{\square}$ is sound 
and complete wrt rooted $G L$-models $\langle K, r\rangle$ of depth 4 , such that every finite subset $X \subseteq K$ of depth at most 2 has a tight predecessor in $K \backslash\{r\}$. (Caveat lector: definition of depth used in [14] is off by one from the present one.)

The idea is as follows. At depth 1 , we have names for each element of $n$, distinguished by the variables $p_{i}$. A subset $a \subseteq n$ is represented by a depth-2 point, having the names for the elements of $a$ as its depth- 1 successors. We use the variable $t$ to indicate whether $a \in X$ or not; the fourth conjunct of $\bar{\Phi}$ ensures that the answer does not depend on the choice of the representant for $a$. The situation is however more complicated as $\varphi$ involves a sequence of sets $a_{0}, \ldots, a_{k-1}$. To this end, we also have indices $j<k$ at depth 1 using atoms $q_{j}$, and each $a \subseteq n$ has several representants at depth 2, labelled by $j<k$. Then we can read off the value of $\varphi(X, \vec{a})$ at a point which has as its successors the representants of $a_{0}, \ldots, a_{k-1}$ with the corresponding labels. (The premise of the last conjunct of $\bar{\Phi}$ reads "for each $j$, I see a unique set labelled by $j$ ".) The important point is that existence of tight predecessors ensures any set has a representant with any label, thus we do not miss any instances of the universal quantifiers in $\Phi$. We proceed with the formal details.

Assume that $n \Vdash \Phi$, and fix a witness $X \subseteq \mathcal{P}(n)$ to the existential quantifier. We construct a model $\langle K, r\rangle$ as follows. Take $n+k$ points of depth 1 , and attach all required tight predecessors, and the root $r$. Define valuation of variables so that each $p_{i}$ or $q_{j}$ is satisfied in one point of depth 1 and nowhere else, with distinct variables getting distinct points, and

$$
u \Vdash t \quad \text { iff } \quad\left\{i<n ; u \Vdash \diamond p_{i}\right\} \in X .
$$

Clearly, the first three conjuncts of $\bar{\Phi}$ are valid in $r$. The fourth one is also valid: if $u \Vdash$ $\bigwedge_{i}\left(\square\left(\diamond \top \rightarrow \diamond p_{i}\right) \vee \square \neg \nabla p_{i}\right)$, all $v>u$ agree on satisfaction of the formulas $\nabla p_{i}$, thus by definition, also agree on satisfaction of $t$.

Assume

$$
u \Vdash \bigwedge_{i, j}\left(\square\left(\diamond q_{j} \rightarrow \diamond p_{i}\right) \vee \square\left(\diamond q_{j} \rightarrow \neg \diamond p_{i}\right)\right) \wedge \bigwedge_{j} \diamond \diamond q_{j},
$$

and put $a_{j}=\left\{i<n ; u \Vdash \diamond\left(\diamond p_{i} \wedge \diamond q_{j}\right)\right\}$. If $u<v \Vdash \diamond q_{j}$, we have

$$
\left\{i<n ; v \Vdash \diamond p_{i}\right\}=a_{j},
$$

as $v \Vdash \diamond p_{i}$ implies $i \in a_{j}$ by definition, and $v \nVdash \diamond p_{i}$ implies $u \nVdash \square\left(\diamond q_{j} \rightarrow \diamond p_{i}\right)$, thus $u \Vdash \square\left(\diamond q_{j} \rightarrow \neg \nabla p_{i}\right)$, and $i \notin a_{j}$.

Therefore $u \Vdash \diamond\left(t \wedge \diamond q_{j}\right)$ implies $a_{j} \in X$, and $u \Vdash \diamond\left(\neg t \wedge \diamond q_{j}\right)$ implies $a_{j} \notin X$; as $u \Vdash \bigwedge_{j} \diamond \diamond q_{j}$, we have

$$
a_{j} \in X \quad \text { iff } \quad u \Vdash \diamond\left(t \wedge \diamond q_{j}\right) .
$$

By definition $i \in a_{j}$ iff $u \Vdash \diamond\left(\diamond p_{i} \wedge \diamond q_{j}\right)$. As $\varphi$ holds for $\vec{a}$, we thus have $u \Vdash \bar{\varphi}$, which completes the verification of $r \Vdash \bar{\Phi}$.

For the converse, assume $\langle K, r\rangle$ is an $A_{L}^{\square}$-model of $\bar{\Phi}$, and define

$$
X=\left\{\left\{i<n ; u \Vdash \diamond p_{i}\right\} ; u \Vdash \square^{2} \perp \wedge \diamond \top \wedge t\right\} .
$$

We claim

$$
u \Vdash t \quad \text { iff } \quad a(u):=\left\{i<n ; u \Vdash \diamond p_{i}\right\} \in X
$$


for any $u$ of depth 2. Indeed, if $u \Vdash t$, then $a(u) \in X$ by definition. If $u \nVdash t$ and $a(u) \in X$, choose $u^{\prime}$ of depth 2 such that $a\left(u^{\prime}\right)=a(u)$, and $u^{\prime} \Vdash t$. Let $v$ be a tight predecessor of the set $\left\{u, u^{\prime}\right\}$. Then $v \Vdash \square\left(\diamond \top \rightarrow \diamond p_{i}\right)$ if $i \in a(u)$, and $v \Vdash \square\left(\diamond \top \rightarrow \neg \nabla p_{i}\right.$ ) (thus $v \Vdash \square \neg \diamond p_{i}$ ) if $i \notin a(u)$. As $v>r \Vdash \bar{\Phi}$, we must have $v \Vdash \square(\diamond \top \rightarrow t)$ or $v \Vdash \square(\diamond \top \rightarrow \neg t)$, which contradicts $u \Vdash \neg t$ or $u^{\prime} \Vdash t$.

Let $a_{0}, \ldots, a_{k-1}$ be arbitrary subsets of $n$, we must show that $\varphi$ holds for $X$ and $\vec{a}$. By the first three conjuncts of $\bar{\Phi}$, we may fix pairwise distinct depth-1 points $x_{i} \Vdash p_{i}$ and $y_{j} \Vdash q_{j}$. For each $j<k$, let $z_{j}$ be a tight predecessor of the set $\left\{y_{j}\right\} \cup\left\{x_{i} ; i \in a_{j}\right\}$, and let $w$ be a tight predecessor of $\left\{z_{j} ; j<k\right\}$. For each $j, w$ has exactly one successor satisfying $\diamond q_{j}$, viz. $z_{j}$. It follows easily

$$
w \Vdash \bigwedge_{i, j}\left(\square\left(\diamond q_{j} \rightarrow \diamond p_{i}\right) \vee \square\left(\diamond q_{j} \rightarrow \neg \diamond p_{i}\right)\right) \wedge \bigwedge_{j} \diamond \diamond q_{j},
$$

thus $w \Vdash \bar{\varphi}$. As we have $w \Vdash \diamond\left(\diamond p_{i} \wedge \diamond q_{j}\right)$ iff $z_{j} \Vdash \diamond p_{i}$ iff $i \in a_{j}$, and $w \Vdash \diamond\left(t \wedge \diamond q_{j}\right)$ iff $z_{j} \Vdash t$ iff $a_{j}=\left\{i ; z_{j} \Vdash \diamond p_{i}\right\} \in X$, this implies $\varphi(X, \vec{a})$.

\subsection{Superintuitionistic logics}

The aim of the present subsection is to prove the following theorem.

Theorem 3.3 Admissibility in any s.i. logic $L \subseteq B D_{3}$ is coNEXP-hard.

In fact, we will show a slightly stronger result:

Theorem 3.4 For any NEXP-language $P$, there is a polynomial-time function $f(w)=$ $\left\langle\alpha_{w}, \delta_{w}\right\rangle$ such that for every $w$,

- if $w \in P$, there is a substitution $\vec{\chi}$ such that $I P C \vdash \alpha_{w}(\vec{\chi})$ and $B D_{2} \nvdash \delta_{w}(\vec{\chi})$,

- if $w \notin P$, then for every $\vec{\chi}$ such that $B D_{3} \vdash \alpha_{w}(\vec{\chi})$, we have $I P C \vdash \delta_{w}(\vec{\chi})$.

Definition 3.5 For the rest of the subsection, we fix $n$ and

$$
\Phi=\exists X \forall a_{0} \ldots \forall a_{k-1} \varphi(X, \vec{a})
$$

as in lemma 3.1 .

We define the following formulas in variables $p_{i}(i<n), q_{j}(j<k), t, s$ :

$$
\begin{aligned}
\beta_{i, j}^{+} & =t \vee s \rightarrow\left(\neg p_{i} \rightarrow t \wedge s\right) \vee \neg q_{j}, \\
\beta_{i, j}^{-} & =t \vee s \rightarrow \neg p_{i} \vee \neg q_{j}, \\
\gamma & =\bigvee_{i} p_{i} \vee \bigvee_{j} q_{j}, \\
\delta & =\bigvee_{i} \neg p_{i} \vee \bigvee_{j} \neg q_{j},
\end{aligned}
$$


and let $\alpha$ be the conjunction of the formulas

$$
\begin{gathered}
\bigwedge_{i<i^{\prime}}\left(p_{i} \rightarrow \neg p_{i^{\prime}}\right) \wedge \bigwedge_{j<j^{\prime}}\left(q_{j} \rightarrow \neg q_{j^{\prime}}\right) \wedge \bigwedge_{i, j}\left(p_{i} \rightarrow \neg q_{j}\right) \\
t \wedge s \equiv \gamma \\
t \vee s \equiv \bigwedge_{i}\left(\left(\neg p_{i} \rightarrow t \wedge s\right) \vee \neg p_{i}\right) \\
\bigwedge_{i, j}\left(\beta_{i, j}^{+} \vee \beta_{i, j}^{-}\right) \rightarrow \bar{\varphi}
\end{gathered}
$$

where $\bar{\varphi}$ is constructed from $\varphi$ as follows. Rewrite $\varphi$ as an equivalent formula using only $\wedge$, $\vee$, and literals, and replace

$$
\begin{aligned}
& a_{j} \in X \text { with } s \rightarrow \neg q_{j} \vee t, \\
& a_{j} \notin X \text { with } t \rightarrow \neg q_{j} \vee s, \\
& i \in a_{j} \text { with } \beta_{i, j}^{+}, \\
& i \notin a_{j} \text { with } \beta_{i, j}^{-} .
\end{aligned}
$$

Lemma 3.6 If $n \vDash \Phi$, then IPC does not admit $\alpha \sim \delta$.

Proof: We use the characterization from theorem 1.6. Let $X$ be a witness for the existential quantifier in $\Phi$. We construct an extensible model $K$ by taking $n+k$ leaves, each forcing $t, s$, and exactly one variable from the list $\vec{p}, \vec{q}$, we add all required tight predecessors (which are bound not to satisfy any $p_{i}$ or $q_{j}$, by persistence), and for any $u$ which is not a leaf, we put

$$
\begin{aligned}
& u \Vdash t \quad \text { iff } \quad \exists a \in X u \Vdash \bigwedge_{i \in a}\left(\neg p_{i} \rightarrow \gamma\right) \wedge \bigwedge_{i \notin a} \neg p_{i}, \\
& u \Vdash s \quad \text { iff } \quad \exists a \notin X u \Vdash \bigwedge_{i \in a}\left(\neg p_{i} \rightarrow \gamma\right) \wedge \bigwedge_{i \notin a} \neg p_{i} .
\end{aligned}
$$

The first three conjuncts of $\alpha$ are easy to verify, and $\delta$ is not valid in the model. Assume $u \Vdash \bigwedge_{i, j}\left(\beta_{i, j}^{+} \vee \beta_{i, j}^{-}\right)$, we have to show $u \Vdash \bar{\varphi}$. Define $a_{j}=\left\{i ; u \Vdash \beta_{i, j}^{+}\right\}$.

\section{Claim 1}

(i) $i \in a_{j} \Rightarrow u \Vdash \beta_{i, j}^{+}$,

(ii) $i \notin a_{j} \Rightarrow u \Vdash \beta_{i, j}^{-}$,

(iii) $a_{j} \in X \Rightarrow u \Vdash s \rightarrow \neg q_{j} \vee t$,

(iv) $a_{j} \notin X \Rightarrow u \Vdash t \rightarrow \neg q_{j} \vee s$.

Proof: $(i)$ is clear. (ii) is also easy: by assumption $u \Vdash \beta_{i, j}^{+}$or $u \Vdash \beta_{i, j}^{-}$, and $u \nVdash \beta_{i, j}^{+}$by definition of $a_{j}$.

(iii): let $u \leq v \Vdash s$. If $v \Vdash t$, we are done. Otherwise there is $a \notin X$ such that $v \Vdash \bigwedge_{i \in a}\left(\neg p_{i} \rightarrow t \wedge s\right) \wedge \bigwedge_{i \notin a} \neg p_{i}$. As $a_{j} \in X$, we have $a \neq a_{j}$. If $i \in a_{j} \backslash a$, we have $v \Vdash \neg p_{i}$; as $u \Vdash \beta_{i, j}^{+}$, also $v \Vdash\left(\neg p_{i} \rightarrow t \wedge s\right) \vee \neg q_{j}$, thus $v \Vdash t \vee \neg q_{j}$. The case $i \in a \backslash a_{j}$ is similar.

$(i v)$ is proved in the same way as (iii), switching $t$ and $s$.

(Claim 1) 
By assumption, $\varphi(X, \vec{a})$ holds. As formulas constructed from $\wedge$ and $\vee$ are monotone, and evaluated in $u$ as in the classical logic, the claim implies $u \Vdash \bar{\varphi}$.

Lemma 3.7 If $B D_{3} \vdash \alpha(\vec{\chi})$ and the image of each $p_{i}$ and $q_{j}$ under $\vec{\chi}$ is $C P C$-consistent, then

$$
n \vDash \exists X \forall \vec{a} \neq \emptyset \varphi(X, \vec{a}) .
$$

Proof: Let $\tilde{\psi}=\psi(\vec{\chi})$ for any $\psi$. Pick single-element models $x_{i} \Vdash \tilde{p}_{i}$ and $y_{j} \Vdash \tilde{q}_{j}$, and construct a depth-3 model $\tilde{K}$ by taking $\vec{x}$ and $\vec{y}$ as leaves, and attaching tight predecessors to all subsets of depth at most 2 . By assumption, $\tilde{K} \Vdash \tilde{\alpha}$. Let $K$ be the model on the same frame as $\tilde{K}$ such that $K, u \Vdash \psi$ iff $\tilde{K}, u \Vdash \tilde{\psi}$.

We have $K \Vdash \alpha$. Notice that $t \wedge s$ is valid in the leaves of $K$, but nowhere else, as every other point of $K$ sees at least two leaves, and thus cannot force any $p_{i}$ or $q_{j}$. Define

$$
\begin{aligned}
& X=\left\{\left\{i ; u \Vdash \neg p_{i} \rightarrow s\right\} ; u \Vdash t, d(u)=2\right\}, \\
& Y=\left\{\left\{i ; u \Vdash \neg p_{i} \rightarrow t\right\} ; u \Vdash s, d(u)=2\right\} .
\end{aligned}
$$

Claim 1 The sets $X$ and $Y$ are disjoint.

Proof: Assume for contradiction that $a \in X \cap Y$, and let $u, u^{\prime}$ be the witnessing points of depth 2 such that $u \Vdash t$ and $u^{\prime} \Vdash s$. Let $v$ be the tight predecessor of $\left\{u, u^{\prime}\right\}$. We have

$$
i \notin a \Rightarrow v \Vdash \neg p_{i},
$$

as $u, u^{\prime} \Vdash \neg p_{i}$ (because $u, u^{\prime} \Vdash t \vee s$ and $u, u^{\prime} \nVdash \neg p_{i} \rightarrow t \wedge s$ ), and $v$ is a t.p. of $\left\{u, u^{\prime}\right\}$. Also

$$
i \in a \Rightarrow v \Vdash \neg p_{i} \rightarrow t \wedge s
$$

Indeed, if $v \leq w \Vdash \neg p_{i}$, then either $v=w$, in which case $u \Vdash t \wedge s$, contradicting $d(u)=2$, or $v<w$, in which case $u \leq w$ or $u^{\prime} \leq w$, thus $w \Vdash t \wedge s$ by $u, u^{\prime} \Vdash \neg p_{i} \rightarrow t \wedge s$.

Thus, as $v \Vdash \alpha$, we get $v \Vdash t \vee s$. This implies $u, u^{\prime} \Vdash t$ or $u, u^{\prime} \Vdash s$, thus $u \Vdash t \wedge s$ or $u^{\prime} \Vdash t \wedge s$, contradicting $d(u)=d\left(u^{\prime}\right)=2$.

$\square$ (Claim 1)

Let $a_{0}, \ldots, a_{k-1}$ be any nonempty sets, we have to show $\varphi(X, \vec{a})$. We have the leaves $x_{i} \Vdash p_{i}$ and $y_{j} \Vdash q_{j}$; let $z_{j}$ be the tight predecessor of $\left\{y_{j}\right\} \cup\left\{x_{i} ; i \in a_{j}\right\}$, and $w$ a t.p. of $\left\{z_{j} ; j<k\right\}$.

\section{Claim 2}

(i) $i \in a_{j} \Rightarrow w \Vdash \beta_{i, j}^{+}$and $w \nVdash \beta_{i, j}^{-}$,

(ii) $i \notin a_{j} \Rightarrow w \nVdash \beta_{i, j}^{+}$and $w \Vdash \beta_{i, j}^{-}$,

(iii) $a_{j} \in X \Rightarrow w \nVdash t \rightarrow \neg q_{j} \vee s$,

(iv) $a_{j} \notin X \Rightarrow w \nVdash s \rightarrow \neg q_{j} \vee t$. 
Proof: If $i \in a_{j}$, then $z_{j} \Vdash \neg p_{i} \rightarrow t \wedge s$ (as $z_{j} \nVdash \neg p_{i}$, and everything strictly above $z_{j}$ has depth 1). If $i \notin a_{j}$, then $z_{j} \Vdash \neg p_{i}$. By $\alpha$ we get $z_{j} \Vdash t \vee s$. Moreover $d\left(z_{j}\right)=2$, thus $z_{j} \nVdash t \wedge s$, and either $z_{j} \Vdash t$ and $a_{j} \in X$, or $z_{j} \Vdash s$ and $a_{j} \in Y$ (thus $a_{j} \notin X$, by claim 1 ).

$(i)$ : as $w \leq z_{j}, z_{j} \Vdash t \vee s$, and $z_{j} \nVdash \neg p_{i} \vee \neg q_{j}$, we have $w \nVdash \beta_{i, j}^{-}$. Consider any $w \leq x \Vdash t \vee s$. We have $x \Vdash \neg p_{i} \rightarrow t \wedge s$ or $x \Vdash \neg p_{i}$. In the latter case, we cannot have $x \leq z_{j}$, thus either $z_{j^{\prime}} \leq x$ for some $j \neq j^{\prime}$, in which case $x \Vdash \neg q_{j}$, or $z_{j}<x$, in which case $x \Vdash t \wedge s$ and a fortiori $x \Vdash \neg p_{i} \rightarrow t \wedge s$.

(iii): we have $w \leq z_{j} \Vdash t, z_{j} \nVdash \neg q_{j} \vee s$.

(ii) and (iv) are proved similarly to (i) and (iii).

(Claim 2)

We have $w \Vdash \bigwedge_{i, j}\left(\beta_{i, j}^{+} \vee \beta_{i, j}^{-}\right)$, thus $w \Vdash \bar{\varphi}$ by $\alpha$. As formulas built from $\wedge$ and $\vee$ are monotone, claim 2 implies $\varphi(X, \vec{a})$.

Proof (theorem 3.4): By lemma 3.1, there is a polynomial-time function which reduces $P$ to validity of $n \vDash \Phi$. WLOG we may further assume

$$
n \vDash \Phi \quad \text { iff } \quad n \vDash \exists X \forall \vec{a} \neq \emptyset \varphi(X, \vec{a}) .
$$

We let $f(w)=\langle\alpha, \delta\rangle$ as in definition 3.5.

If $n \vDash \Phi$, there is a substitution $\vec{\chi}$ such that IPC $\vdash \alpha(\vec{\chi})$ and IPC $\nvdash \delta(\vec{\chi})$ by lemma 3.6. As $\delta$ is a disjunction of negated formulas, each disjunct has a classical (i.e., depth-1) countermodel, and the whole disjunction has a depth-2 countermodel, thus $B D_{2} \nvdash \delta(\vec{\chi})$.

If $n \not \models \Phi$ and $\vec{\chi}$ is such that $B D_{3} \vdash \alpha(\vec{\chi})$, one of the disjuncts of $\delta(\vec{\chi})$ is provable in $C P C$ by lemma 3.7. As the disjuncts are negative, we have $I P C \vdash \delta(\vec{\chi})$.

Theorem 3.3 gives a simple class of logics for which our method works, but the lower bound is applicable also to other systems. Most importantly, the condition $L \subseteq B D_{3}$ excludes logics whose frames have a single top point, such as $K C$. We note that admissibility in $K C$ is also coNEXP-hard, though we do not know how to generalize the result to, say, all logics contained in $\mathrm{KC}+\mathrm{BD}_{4}$.

Lemma 3.8 Let $\varphi(q, \vec{p})$ and $\psi(q, \vec{p})$ be $\perp$-free formulas with all variables shown, and $r$ a new variable.

(i) IPC $\vdash \varphi(\perp, \vec{p})$ iff IPC $\vdash \varphi\left(\bigwedge_{i} p_{i} \wedge r, \vec{p}\right)$.

(ii) $\varphi(\perp, \vec{p}) \sim_{I P C} \psi(\perp, \vec{p})$ iff $\varphi\left(\bigwedge_{i} p_{i} \wedge r, \vec{p}\right) \sim_{I P C} \psi\left(\bigwedge_{i} p_{i} \wedge r, \vec{p}\right)$.

(iii) If $\varphi(\vec{p}) \mathcal{W}_{I P C} \psi(\vec{p})$, there exists a $\perp$-free substitution $\vec{\chi}$ such that IPC $\vdash \varphi(\vec{\chi})$, and $I P C \nvdash \psi(\vec{\chi})$.

Proof: $(i)$ is a special case of $(i i)$.

(ii): the right-to-left implication follows from the substitution $r \mapsto \perp$. Let $\langle K, \leq, \Vdash\rangle$ be an extensible model which validates $\varphi\left(\bigwedge_{i} p_{i} \wedge r, \vec{p}\right)$, and refutes $\psi\left(\bigwedge_{i} p_{i} \wedge r, \vec{p}\right)$ in $x \in K$. Put $K^{\prime}=\left\{u \in K ; u \nVdash \bigwedge_{i} p_{i} \wedge r\right\}$. $K^{\prime}$ is extensible: t.p.'s of nonempty sets exist as $K^{\prime}$ is downwards closed, and the empty set has a t.p. as $K^{\prime} \ni x$ is nonempty. Moreover, $K^{\prime}$ validates $\varphi(\perp, \vec{p})$, and $K^{\prime}, x \nVdash \psi(\perp, \vec{p})$.

(iii): take $\perp$-free formulas $\vec{\chi}$ such that $I P C \vdash \varphi(\vec{\chi}(\perp, \vec{q}))$ and $\operatorname{IPC} \nvdash \psi(\vec{\chi}(\perp, \vec{q}))$. Then $I P C \vdash \varphi\left(\vec{\chi}\left(\bigwedge_{i} q_{i} \wedge r, \vec{q}\right)\right)$ and $I P C \nvdash \psi\left(\vec{\chi}\left(\bigwedge_{i} q_{i} \wedge r, \vec{q}\right)\right)$ by $(i)$. 
Proposition 3.9 Admissibility in KC is coNEXP-hard.

Proof: Let $f(\varphi(\perp, \vec{p}) \triangleright \psi(\perp, \vec{p}))=\varphi\left(\bigwedge_{i} p_{i} \wedge r, \vec{p}\right) \triangleright \psi\left(\bigwedge_{i} p_{i} \wedge r, \vec{p}\right)$, we claim that $f$ is a reduction of $I P C$-admissibility to $K C$-admissibility.

If $\varphi(\perp, \vec{p}) \sim_{I P C} \psi(\perp, \vec{p})$, then $\varphi\left(\bigwedge_{i} p_{i} \wedge r, \vec{p}\right) \sim_{I P C} \psi\left(\bigwedge_{i} p_{i} \wedge r, \vec{p}\right)$ by lemma 3.8. As $K C$ admits (single-conclusion) Visser's rules [12], this implies $\varphi\left(\bigwedge_{i} p_{i} \wedge r, \vec{p}\right) \sim_{K C} \psi\left(\bigwedge_{i} p_{i} \wedge r, \vec{p}\right)$.

Assume $\varphi(\perp, \vec{p}) \mathcal{W}_{I P C} \psi(\perp, \vec{p})$. Then $\varphi\left(\bigwedge_{i} p_{i} \wedge r, \vec{p}\right) \mathcal{W}_{I P C} \psi\left(\bigwedge_{i} p_{i} \wedge r, \vec{p}\right)$, thus there exist $\perp$-free formulas $\vec{\chi}$ and $\varrho$ such that $I P C \vdash \varphi\left(\bigwedge_{i} \chi_{i} \wedge \varrho, \vec{\chi}\right)$, and $I P C \nvdash \psi\left(\bigwedge_{i} \chi_{i} \wedge \varrho, \vec{\chi}\right)$. This implies $\varphi\left(\bigwedge_{i} p_{i} \wedge r, \vec{p}\right) \mathcal{W}_{K C} \psi\left(\bigwedge_{i} p_{i} \wedge r, \vec{p}\right)$, because $K C$ has the same $\perp$-free fragment as $I P C$ $($ see $[5])$.

\subsection{Modal logics}

Definition 3.10 T is Gödel's translation of $I P C$ in modal logic, adjusted to nonreflexive extensions of $K 4: \mathrm{T}(p)=\square p$ for atoms $p, \mathbf{T}(\perp)=\perp, \mathrm{T}(\varphi \circ \psi)=\mathrm{T}(\varphi) \circ \mathrm{T}(\psi)$ for $\circ \in\{\wedge, \vee\}$, and $\mathrm{T}(\varphi \rightarrow \psi)=\square(\mathrm{T}(\varphi) \rightarrow \mathrm{T}(\psi))$.

If $L$ is an extension of $K 4, \varrho L$ is the s.i. $\operatorname{logic}\{\varphi ; L \vdash \mathrm{T}(\varphi)\}$. For any transitive frame $K$, its skeleton $\varrho K$ is the intuitionistic frame consisting of the clusters of $K$ with the induced ordering relation.

We will transfer the result of the previous subsection to the modal case rather easily, using Gödel's translation. However, we need to formulate the assumptions a bit more carefully; the condition $L \subseteq K 4 B D_{3}$ is too strong (it excludes e.g. $S 4$ and $G L$ ), whereas $\varrho L \subseteq B D_{3}$ seems too weak. The next definition says, roughly, that every depth-3 tree is a skeleton of an $L$ frame, in which the final clusters are prescribed by the enemy along with the skeleton, but we are free to chose the clusters at depths 2 and 3.

Definition 3.11 Let $L$ be a consistent normal extension of $K 4 . \quad L$ has the weak depth-3 extension property, if for every finite tree $T$ of depth 3 whose leaves are labeled by finite disjoint clusters validating $L$, there is an $L$-frame $K$ such that $\varrho K \simeq T$, and the preimage of any leaf of $T$ under the $\varrho$ construction is its label.

Notice that we may wlog require all clusters in $K$ of depth 2 and 3 to be singletons, and state the property for arbitrary finite directed graphs of depth 3 instead of trees. Both observations follow as the class of $L$-frames is closed under p-morphisms.

Lemma 3.12 Let $L$ be a normal extension of $K 4$. If $\square \varphi$ is L-consistent, it is satisfiable in an L-frame which is a finite cluster.

Proof: If $\square \varphi$ is consistent with $L \oplus(S 5 \cap(K \oplus \square \perp))=(L \oplus S 5) \cap(L \oplus \square \perp)$, we are done, as all extensions of $S 5$ (Bull [2]) or $K \oplus \square \perp$ (trivial) have FMP. If not, there is $\psi$ such that $L \vdash \psi$ and $S 5 \cap(K \oplus \square \perp) \vdash \psi \rightarrow \diamond \neg \varphi$. We have

$$
S 5 \cap(K \oplus \square \perp) \vdash \alpha \Rightarrow K 4 \vdash \diamond \alpha
$$

for any $\alpha$ : otherwise $\square \neg \alpha$ is satisfiable in a finite $K 4$-model, thus in a final cluster, which is a model of $S 5 \cap(K \oplus \square \perp)$. 
Therefore $K 4 \vdash \diamond(\psi \rightarrow \diamond \neg \varphi)$ and $L \vdash \diamond \neg \varphi$, a contradiction.

Theorem 3.13 If $L$ is a normal extension of $K 4$ with the weak depth-3 extension property, then admissibility in $L$ is coNEXP-hard.

Proof: Consider the reduction from theorem 3.4. We claim

$$
w \notin P \quad \text { iff } \quad \mathbf{T}\left(\alpha_{w}\right) \sim_{L} \mathbf{T}\left(\delta_{w}\right) .
$$

If $w \in P$, there is a substitution $\vec{\chi}$ such that $I P C \vdash \alpha(\vec{\chi})$, and $B D_{2} \nvdash \delta(\vec{\chi})$. Then $L \vdash \mathrm{T}(\alpha)(\mathrm{T}(\vec{\chi}))$ and $L \nvdash \mathrm{T}(\delta)(\mathrm{T}(\vec{\chi}))$, as $\mathrm{T}$ commutes with substitution (up to $K 4$-provable equivalence), and $\varrho L \subseteq B D_{3} \subseteq B D_{2}$ by the weak depth-3 extension property.

Let $\vec{\chi}$ be a substitution such that $L \vdash \mathrm{T}(\alpha)(\vec{\chi})$ and $L \nvdash \mathrm{T}(\delta)(\vec{\chi})$. Put $\tilde{\psi}=\mathrm{T}(\psi)(\vec{\chi})$ for any intuitionistic formula $\psi$. As $\tilde{\delta}=\bigvee_{i} \square \neg \tilde{p}_{i} \vee \bigvee_{j} \square \neg \tilde{q}_{j}$, the formulas $\tilde{p}_{i}$ and $\tilde{q}_{j}$ are $L$-consistent. As these formulas have the form $\square(\ldots)$, there are finite clusters $C_{i} \Vdash \tilde{p}_{i}$ and $D_{j} \Vdash \tilde{q}_{j}$ validating $L$, by lemma 3.12. By the weak depth-3 extension property, there is an $L$-model $K$ of depth 3 which has $\vec{C}$ and $\vec{D}$ as its final clusters, and contains a tight predecessor (either reflexive or irreflexive) for every subset of depth at most 2. By assumption, $K \Vdash \tilde{\alpha}$. Define a valuation on $\varrho K$ by $[u] \Vdash \psi$ iff $u \Vdash \tilde{\psi}$. Then $\varrho K$ is a model of the form considered in the proof of lemma 3.7, thus by the same argument, $n \Vdash \Phi$ and $w \notin P$.

The $\mathrm{T}$ translation is not in general polynomial-time: expanding each subformula $\square \varphi=$ $\varphi \wedge \square \varphi$ doubles the size, thus the length of $T(\varphi)$ is exponential in the implication-depth of $\varphi$. However, our $\alpha$ and $\delta$ from definition 3.5 have constant depth (4), which takes care of the problem. (In fact, it is easy to see that any rule can be transformed into a polynomially longer rule involving only formulas of depth 2, while preserving its (non)admissibility.)

Corollary 3.14 Admissibility in any extensible finitely axiomatizable cofinal subframe logic is coNEXP-complete.

Notice that the constant 3 in theorem 3.13 is tight: $L=G L+\square^{2} \perp$ has the depth2 extension property, yet by proposition 2.7, $L$-admissibility is not coNEXP-hard (unless NEXP , including the polynomial-time hierarchy, collapses to $\Sigma_{3}^{P}=\Pi_{3}^{P}$ ). In fact, we have the following characterization, which illustrates the dependence of complexity of admissibility on depth. Notice that derivability in any of the logics $G L+\square^{m} \perp$ is coNP-complete.

Proposition 3.15 Let $m>0$. Admissibility in $G L+\square^{m} \perp$ is

(i) coNP-complete for $m=1$,

(ii) $\Pi_{3}^{P}$-complete for $m=2$,

(iii) coNEXP-complete for $m>2$.

Proof: (i): $G L+\square \perp$ is an inessential variant of $C P C$.

(iii) follows from theorem 3.2.

(ii): $A_{L}^{\square} \in \Pi_{3}^{P}$ by proposition 2.7. We can reduce validity of $\Sigma_{3}^{q}$ quantified Boolean formulas to $L$-nonadmissibility as in the proof of theorem 3.2, using tight predecessors to simulate universal quantifiers. Details are left to the interested reader. 


\section{Acknowledgement}

I wish to thank Albert Visser for asking the right question at the right time, and the anonymous referee for useful suggestions.

\section{References}

[1] Patrick Blackburn, Maarten de Rijke, and Yde Venema, Modal logic, Cambridge Tracts in Theoretical Computer Science vol. 53, Cambridge University Press, 2001.

[2] Robert A. Bull, That all normal extensions of S4.3 have the finite model property, Zeitschrift für mathematische Logik und Grundlagen der Mathematik 12 (1966), pp. 341-344.

[3] Alexander V. Chagrov, On the complexity of propositional logics, in: Complexity problems in Mathematical Logic, Kalinin State University, 1985, pp. 80-90 (in Russian).

[4] _ A decidable modal logic with the undecidable admissibility problem for inference rules, Algebra and Logic 31 (1992), pp. 53-55.

[5] Alexander V. Chagrov and Michael Zakharyaschev, Modal logic, Oxford Logic Guides vol. 35, Oxford University Press, 1997.

[6] Ronald Fagin, Generalized first-order spectra and polynomial-time recognizable sets, in: Complexity of Computation (R. M. Karp, ed.), SIAM-AMS Proceedings vol. 7, 1974, pp. $43-73$.

[7] Harvey M. Friedman, One hundred and two problems in mathematical logic, Journal of Symbolic Logic 40 (1975), no. 2, pp. 113-129.

[8] Silvio Ghilardi, Unification in intuitionistic logic, Journal of Symbolic Logic 64 (1999), no. 2 , pp. $859-880$.

[9] _ Best solving modal equations, Annals of Pure and Applied Logic 102 (2000), no. 3, pp. 183-198.

[10] A resolution/tableaux algorithm for projective approximations in IPC, Logic Journal of the IGPL 10 (2002), no. 3, pp. 229-243.

[11] Rosalie Iemhoff, On the admissible rules of intuitionistic propositional logic, Journal of Symbolic Logic 66 (2001), no. 1, pp. 281-294.

[12] Intermediate logics and Visser's rules, Notre Dame Journal of Formal Logic 46 (2005), no. 1, pp. 65-81.

[13] On the rules of intermediate logics, Archive for Mathematical Logic 45 (2006), no. 5, pp. 581-599. 
[14] Emil Jeřábek, Admissible rules of modal logics, Journal of Logic and Computation 15 (2005), no. 4, pp. 411-431.

[15] Alexander V. Kuznetsov, On superintuitionistic logics, in: Proceedings of the International Congress of Mathematicians (Vancouver), 1975, pp. 243-249.

[16] Richard E. Ladner, The computational complexity of provability in systems of modal propositional logic, SIAM Journal on Computing 6 (1977), no. 3, pp. 467-480.

[17] Christos H. Papadimitriou, Computational complexity, Addison-Wesley, 1994.

[18] Vladimir V. Rybakov, Admissibility of logical inference rules, Studies in Logic and the Foundations of Mathematics vol. 136, Elsevier, 1997.

[19] Edith Spaan, Complexity of modal logics, Ph.D. thesis, University of Amsterdam, 1993.

[20] Richard Statman, Intuitionistic propositional logic is polynomial-space complete, Theoretical Computer Science 9 (1979), no. 1, pp. 67-72.

[21] Michael Zakharyaschev, Canonical formulas for K4. Part II: Cofinal subframe logics, Journal of Symbolic Logic 61 (1996), no. 2, pp. 421-449. 